\title{
EFECTOS DEL CAPITAL SOCIAL EN EL EMPLEO EN MÉXICO*
}

\author{
Humberto Charles-Leija \\ Aldo Josafat Torres Garcia ${ }^{\mathrm{b}}$ \\ David Castro Lugo
}

* DOI: https://doi.org/10.18601/01245996.v20n38.11. Este artículo es resultado de la investigación Mercado laboral en México: mecanismos de búsqueda, movilidad y desigualdad salarial, apoyado por la Coordinación General de Investigación y Posgrado de la Universidad Autónoma de Coahuila. Recepción: 19-12-2016, modificación: 22-01-2018, aceptación: 19-02-2018. Sugerencia de citación: Charles L., H.; Torres G., A. J. y Castro L., D. (2018). Efectos del capital social en el empleo en México. Revista de Economía Institucional, 20(38), 263-283.

a Doctor en Economía Regional. Profesor del Instituto Tecnológico de Saltillo, México, [humbertocharles@yahoo.com].

b Doctor en Economía Regional. Profesor de la Universidad Autónoma de Chihuahua, México, [aldo.torres@uadec.edu.mx].

c Doctor en Economía Aplicada. Coordinador de los posgrados en Economía Regional del Centro de Investigaciones Socioeconómicas de la Universidad Autónoma de Coahuila, México, [david.castro@uadec.edu.mx]. 


\section{Efectos del capital social en el empleo en México}

Resumen. Este artículo explora la influencia de las redes sociales físicas y electrónicas en el mercado laboral, empleando datos de la Encuesta de Bienestar Autorreportado para México en 2014. El capital social ejerce una influencia positiva en la situación laboral de los individuos. Tener más de tres amigos disminuye la probabilidad de estar desempleado entre el 1,1 y el 1,8\%; mientras que la red familiar y las redes sociales electrónicas no resultan significativas. Los resultados sugieren que el capital familiar es una especie de "seguro de desempleo"y que los amigos proporcionan información sobre vacantes y salarios.

Palabras clave: capital social, redes sociales, Facebook, Twitter, internet, búsqueda de empleo; JEL: J64, Z13

\section{The effects of social capital on employment in Mexico}

Abstract. This article explores the influence of physical and electronic social networks on the labor market, using data from the Self-Reported Welfare Survey for Mexico in 2014. Social capital has a positive influence on the employment situation of individuals. Having more than three friends decreases the probability of being unemployed between 1,1 and 1,8\%; while the family network and electronic social networks are not significant. The results suggest that family capital is a kind of "unemployment insurance" and that friends provide information about vacancies and salaries.

Keywords: social capital, social networks, Facebook, Twitter, internet, job search; JEL: J64, Z13

\section{Efeitos do capital social no emprego no México}

Resumo. Este artigo explora a influência das redes sociais físicas e eletrônicas no mercado de trabalho, utilizando dados da Pesquisa de Bem-estar Auto-projetado (Encuesta de Bienestar Autorreportado) para o México, em 20140, O capital social exerce uma influência positiva na situação de trabalho dos indivíduos0, Possuir mais de três amigos diminui a probabilidade de estar desempregado entre 1,1 e $1,8 \%$; enquanto que a rede familiar e as redes sociais eletrônicas não parecem ser significativas0, Os resultados sugerem que o capital familiar é uma espécie de "seguro desemprego" e que os amigos proporcionam informação sobre vagas e salários.

Palavras-chaves: capital social, redes sociais, Facebook, Twitter, Internet, procura de emprego; JEL: J64, Z13 
- 1 desempleo genera efectos económicos y sociales negativos, Cdesde reducciones del bienestar individual y pérdidas de capital humano hasta aumentos de la tasa de criminalidad (Winkelmann y Winkelmann, 1998; Lin, 2008). Desde hace más de cuatro décadas, algunos estudios han señalado la relevancia de los canales informales como opciones para encontrar empleo (Rees, 1966; Rees y Schultz, 1970; Reid, 1972). En años recientes continúa el interés por identificar si los canales informales siguen siendo mecanismos relevantes para encontrar empleo frente a mecanismos alternativos (Bachmann y Baumgarten, 2012; Cappellari y Tatsiramos, 2010).

Este artículo se pregunta cuál es la contribución de la red de contactos a la probabilidad de obtener empleo. Para responder esa pregunta aborda inicialmente uno de los elementos que, a nivel individual, favorece la salida del desempleo: el capital social.

En lo referente a búsqueda de empleo, la investigación reciente se enfoca en mecanismos informales como las redes de amigos y familiares (Rees, 1966). Diversos estudios exploran este tema (Montgomery, 1991; Calvó-A. y Jackson, 2005; Calvó-A. e Ioannides, 2005). A diferencia de ellos, el presente trabajo hace un acercamiento al uso de redes sociales electrónicas y evalúa la probabilidad de desempleo que tienen los individuos a partir de su capital social.

En México, la Encuesta de Bienestar Autorreportado (BIARE) del Instituto de Estadística y Geografía (INEGI) indica que una mayor red de amistades disminuye la posibilidad de que los individuos estén desempleados cuando tienen más de tres amigos. No ocurre lo mismo en cuanto a los familiares, quizá porque el apoyo que proporcionan está más relacionado con aspectos emocionales el hecho de compartir bienes durables. Las redes sociales-Facebook, Twitter y otras- no son significativas para la probabilidad de obtener empleo.

$\mathrm{E} 1$ artículo se divide en cuatro secciones. En la primera se describen los planteamientos teóricos acerca de la utilización del capital social en el mercado laboral; la segunda describe la metodología y la hipótesis que se propone; en la tercera se presentan los resultados y al final se exponen las principales conclusiones.

\section{PLANTEAMIENTOS TEÓRICOS}

En el mercado laboral ocurre un emparejamiento entre firmas y trabajadores. Cuando los empresarios no tienen completa su plantilla de trabajadores inician un proceso para reclutar candidatos adecuados; asimismo, cuando un individuo quiere encontrar un empleo se da a la tarea de buscar una vacante que le ofrezca salario y condiciones 
favorables (Petrongolo y Pissarides, 2001). Las firmas determinan con qué intensidad intentarán cubrir una vacante, considerando costo y tiempo de la búsqueda, características del puesto y tratando de encontrar al candidato idóneo. Por su parte, los desempleados controlan la intensidad de su búsqueda y a quién le comunican su necesidad de empleo; asimismo, los empleadores promueven la difusión de la vacante entre posibles aspirantes (Diamond, 1982).

Ahora bien, tanto empleadores como buscadores de empleo incurren en costos durante el periodo en que aún no se ha dado el emparejamiento. Los empleadores cuentan con una vacante al interior de la firma, en la que falta alguien que le genere beneficios a la empresa; asimismo, el desempleado se enfrenta a un coste de oportunidad por los días que busca empleo en lugar de producir para una firma y obtener un salario (Petrongolo y Pissarides, 2001).

Mortensen (1982) sugirió que los incentivos que tienen los agentes para lograr el emparejamiento en el mercado laboral dependen de los costos en que incurren por no alcanzarlo; es decir, si un individuo es jefe de hogar y requiere un ingreso laboral para la subsistencia de la familia tendrá incentivos para encontrar un empleo pronto; de la misma manera, una empresa que requiere de una plantilla de operarios para la producción diaria tendrá incentivos para cubrir las vacantes con prontitud. El presente documento se enfoca en el lado del buscador de empleo.

\section{Búsqueda del mejor empleo a partir del capital personal disponible}

Las firmas buscan los empleados más calificados para sus vacantes, aquellos que mayor valor agregado puedan generar para la empresa. Mientras los individuos buscan empleos que ofrezcan el salario por hora más alto posible, así como las mejores condiciones laborales en cuanto a seguridad física, estabilidad, desarrollo laboral y prestaciones.

Para asegurarse la mejor oferta laboral, los trabajadores invierten en capital humano, social e informativo. Lo cual les permite ser más productivos y tener información relevante sobre el mercado laboral. En este sentido podría establecerse que una persona cuenta con capital humano (educación, experiencia, etc.); capital social, conformado por las redes sociales que posee; y capital informativo, es decir, conocimiento sobre el mercado laboral (tasas salariales y condiciones laborales).

La inversión en capital humano son las actividades que pretenden mejorar las capacidades físicas y mentales de las personas con 
la expectativa de incrementar los ingresos, de manera inmediata o posterior (Becker, 1962).

El capital social ${ }^{1}$ refiere a las conexiones y lazos de apoyo recíproco que tienen las personas. Se piensa que las redes de capital social pueden repercutir en beneficios de índole económica y de seguridad ciudadana (Helliwell y Putnam 2004). Adicional a la relevancia del capital social es importante la velocidad con la que se hace uso de las redes. Calvó-A. y Jackson (2004) sugieren que las redes sociales se debilitan al pasar mucho tiempo en situación de desempleo.

El presente documento evalúa el capital social ${ }^{2}$ a partir de dos conceptos: redes sociales físicas y redes sociales electrónicas (o virtuales). La primera definida por el conjunto de contactos, familiares, amigos o conocidos, que tiene un individuo y que le permite recibir apoyo, pudiendo este ser de tipo emocional, financiero, moral, etc.; mientras las segundas refieren al entorno virtual, es decir, contactos, familiares, amigos o conocidos localizables a través de internet, tales como Facebook, Twitter, Whatsapp, etc.

Es importante hacer esta distinción porque las redes sociales virtuales pueden ser más débiles que las redes sociales físicas, basadas en relaciones más sólidas.

Stigler (1962) señaló que la información que un individuo posee respecto al mercado de trabajo puede entenderse como capital. Para obtener este capital de información es necesario invertir tiempo y dinero en la búsqueda de información. Entre mejor informada esté una persona, más próxima estará a maximizar su salario. Así, la posibilidad de recibir información de parte de amigos y familiares permite disponer de más herramientas para obtener mejores condiciones laborales. Las relaciones sociales son un apoyo para los desempleados en su búsqueda de trabajo, así como para los empleadores en su búsqueda de personal (Calvó-A. y Ioannides, 2005).

\section{Canales de búsqueda de empleo}

Rees (1966) dividió las redes de información en dos tipos: formales e informales. Las redes formales incluyen servicios de búsqueda de empleo estatales, agencias de colocación, avisos de prensa, reuniones sindicales y departamentos escolares de vinculación. Los mecanismos

\footnotetext{
${ }^{1}$ Aquí nos referimos al capital social en sentido individual, no de comunidad como es usual en la literatura.

${ }^{2} \mathrm{Si}$ bien algunos autores han sugerido que las habilidades sociales son un complemento del capital humano (Glaeser et al., 2002), el trabajo actual entiende al factor social como capital en sí mismo.
} 
informales corresponden a referencias de personas empleadas y a visitas directas a los establecimientos. En los niveles de calificación altos, los mecanismos informales explicaban la mitad de las contrataciones y en niveles de calificación bajos el porcentaje era aún mayor. En este trabajo se entiende que los mecanismos formales están ligados a conocimientos que están al alcance del público, mientras que los informales son de carácter más privado y de acceso limitado

Mediante los esquemas informales, los desempleados interrogan a amigos y familiares que tienen empleo sobre las condiciones laborales de los establecimientos donde trabajan, lo que les proporciona mayor información y, si existe una vacante, decidir si la acepta. Las personas empleadas que se enteran de ofertas laborales avisan a sus amigos, empleados y desempleados. Los empleados informan a quien busca trabajo cuales son las responsabilidades del cargo vacante: así, las personas recomendadas pueden evaluar mejor si ese cargo concuerda con sus habilidades y capacidades.

Las personas solo recomiendan a quienes consideran idóneos para los puestos disponibles, y una vez contratados pueden ejercer presión para incentivarlos a esforzarse: un esquema de monitoreo de bajo costo para la firma (Kugler, 2003). Del mismo modo, se ha sugerido que las firmas contratan a parientes de sus empleados porque cuentan con información sobre habilidades, capacidades y carácter de sus trabajadores (Becker, 1981). De esta manera, tanto buscadores de empleo como empleadores se benefician de contar con una persona que funciona como enlace entre unos y otros. Los recomendados ${ }^{3}$ tienen acceso a mayores vacantes disponibles y características relevantes de estas, mientras las firmas cuentan con conocimiento sobre las características de los candidatos. Asimismo, la persona que fungió como enlace entre desempleados y empleadores puede monitorear el desempeño del recomendado en caso de que acceda a una posición en la empresa.

Algunos autores han indagado en la calidad de las conexiones sociales, sugiriendo que entre mayor calidad de empleo tengan los contactos, mejores condiciones tendrán las ofertas laborales que puedan comunicar (Calvó-A- y Jackson, 2005; Bentolila et al., 2003; Aguilera, 2002).

Por su parte, Kugler (2003) argumentó que las referencias empatan trabajadores bien conectados con empleos de alta remuneración, mien-

\footnotetext{
${ }^{3}$ En este trabajo se considera que recomendar a una persona no implica corrupción, es simplemente comunicar al empleador las características laborales del conocido interesado en la vacante. El empleador evalúa al total de candidatos posibles y considera sus referencias.
} 
tras que los medios formales lo hacen entre empleados pobremente conectados y trabajos de menor ingreso.

Aguilera y Massey (2003) identificaron que, para los inmigrantes mexicanos en Estados Unidos, el capital social favorece la posibilidad de encontrar un empleo; sin embargo, al diferenciar por cercanía familiar se encontró que la familia "cercana" ${ }^{4}$ no ejerce un efecto positivo sobre la posibilidad de encontrar empleo, mientras que la familia "lejana" sí. Ello es relevante en el sentido de que la familia más próxima puede ser un apoyo en situación de desempleo y brindar a los individuos un soporte económico y emocional al realizar la búsqueda laboral. Mientras que la familia lejana y amigos pueden ofrecer menores apoyos monetarios pero mayor información sobre vacantes y opciones laborales. Sin embargo, convendría señalar que puede haber un efecto de tamaño, es decir, la familia cercana involucra pocas personas y la lejana un conjunto mayor. Así, la familia lejana es una red más amplia, con acceso a más información sobre vacantes y entorno laboral.

Montgomery (1991) señaló que a finales de la década de 1980 el 50\% de los trabajadores en activo había encontrado su empleo a través de amigos y familiares. La evidencia sugiere que en los sectores de salarios altos los empleadores prefieren basarse en redes sociales personales para llenar sus vacantes (Kugler, 2003). Mientras que para sectores o puestos de baja remuneración se recurre a los esquemas formales, de acceso más sencillo para personas poco conectadas (Wial, 2008).

Si bien la mayoría de los autores que han abordado el tema sugieren efectos favorables de las redes sociales sobre el mercado laboral, también se ha planteado que si los contactos personales tienen menor nivel de calificación que la persona desempleada pueden recomendarle en empleos de menor remuneración que la esperada; a partir de ello, su productividad no se maximizaría y el capital social podría jugar en su contra (Bentolila et al., 2003).

De esta manera, la literatura sugiere que existe una conveniencia de recurrir a una persona enlace que contacte a empleadores y buscadores de empleo. Para los desempleados, el contar con familiares o amigos que cumplan esa función es un canal significativo para acceder a un empleo. Otro canal que puede tener implicaciones destacables es internet; en la siguiente subsección se aborda ese tema.

${ }^{4}$ Cónyuge, hermanos, padres y abuelos.

${ }^{5}$ Tíos, primos, sobrinos y familia política.

Revista de Economía Institucional, vol. 20, N. ${ }^{\circ} 38$, Primer semestre/2oi8, pp. 263-283 


\section{E1 uso de internet para obtener información y fortalecer las redes}

En la actualidad existe elevada disponibilidad de información debido a la existencia de internet y las redes sociales electrónicas. Así, se puede señalar que los costos asociados a la búsqueda laboral se han abaratado en los años recientes; internet permite la difusión de ideas, conocimientos e información de manera casi instantánea, y en nuestros días es posible intercambiar información prácticamente sin costo.

Las firmas y los trabajadores se localizan cerca porque ello reduce los costos de transporte e involucra beneficios para ambos. Sin embargo, la tecnología ha venido reduciendo los costos referentes al traslado de personas, bienes e incluso ideas. En la actualidad algunos autores auguran una "muerte de la distancia" (Ioannides, Overman, Rossi-H. et al., 2008). El uso de internet simplifica la difusión de información e ideas. Permite la comunicación con amigos, familiares, colegas, proveedores y clientes. La transmisión de la información puede emplearse para la búsqueda u ofrecimiento de empleo, debido a sus costos relativamente bajos.

En un estudio sobre Alemania, Mang (2012) identificó que aquellas personas que encontraron un nuevo empleo a través de internet empataron mejor sus capacidades con la nueva posición que si lo hubieran hecho por canales tradicionales como periódicos, amigos o agencias de empleo. Tal situación mejora las posibilidades incluso de habitantes de entornos rurales, quienes salvan la distancia con el mercado laboral gracias al uso de medios electrónicos. Por su parte, Kuhn y Mansour (2014) identificaron mayor probabilidad de encontrar empleo a través del uso de internet, para la población joven de Estados Unidos, observando además un moderado incremento salarial. Señalaron que el porcentaje de jóvenes que buscan empleo por medio de internet creció del 24 al $74 \%$ entre 2000 y 2009. Para el caso de México, Torres y Félix (2017) encontraron que es mayor la probabilidad de un usuario de internet de tener un mayor salario frente a la de aquellos individuos que no son partícipes de esta tecnología. Asimismo, los autores mencionados constataron la existencia de una prima salarial al uso de internet de más del 20\%, y que era creciente a medida que aumentaba el ingreso laboral. Sin embargo, en México, menos del 2\% de los trabajadores activos habían obtenido empleo a través de internet en 2014 (Charles-L., s.f.).

Dicho hallazgo pudiera pensarse que corresponde principalmente a individuos de mayor calificación; sin embargo, desde hace algunos 
años ciertas empresas difunden sus avisos de vacantes a través de redes sociales, a las que un segmento de la población de baja calificación (jóvenes principalmente) tiene acceso.

En la actualidad existe una amplia gama de redes sociales que permiten a los individuos mantenerse en contacto con familiares, amigos y colegas, así como buscar empleo.

Un propósito del uso de Facebook puede ser el de mantener lazos fuertes de amistad con amigos y familiares (Valenzuela, Park y Kee, 2009); la red contribuye a mantener e incrementar el capital social (Ellison et al., 2007; Pénard et al., 2013). Por su parte, Twitter es una red diferente, es un servicio de microblogging ${ }^{6}$ enfocado menos en el fortalecimiento de redes y más en la difusión de ideas y noticias; para seguir publicaciones de otra persona no se requiere necesariamente un lazo afectivo (Kwak et al., 2010). Existen otras redes sociales usadas por los mexicanos, tales como Linkedin, Google plus y Myspace, entre otras. Linkedin tiene un enfoque profesional y se encamina a fortalecer el contacto entre colegas; dicha red pudiera resultar en una herramienta adecuada de búsqueda de empleo para profesionales. Las demás redes son semejantes a Facebook.

Debido a la disponibilidad de datos, este artículo se centra en los individuos que declararon tener cuenta en Facebook, Twitter u "otra red social". En la siguiente sección se describe con más detalle la metodología.

La revisión de la literatura de esta sección muestra que en México son aún recurrentes los mecanismos informales. Y que ahora el uso de redes sociales electrónicas puede facilitar la difusión de información y el fortalecimiento de lazos afectivos. A partir de lo anterior es importante aprovechar las bases de datos que permitan contrastar información sobre redes sociales físicas y virtuales que puedan permitir a los individuos acceder a ofertas laborales; la próxima sección se enfoca en ese aspecto.

\section{METODOLOGÍA Y DATOS}

E1 modelo planteado en el estudio indaga si las redes sociales físicas y electrónicas disminuyen las probabilidades de encontrarse en situación de desempleo. Se formula la propuesta siguiente:

Desempleo $=f($ fam, amig, facebook, twitter, otra red social, controles $)$

${ }^{6}$ Un blog es un sitio web de publicación de textos o artículos de opinión. 
Para contrastar la ecuación (1) se elaboró un modelo logit binomial ${ }^{7}$ con datos de la encuesta BIARE. Si en dicho modelo se obtiene 1 el individuo está desempleado. Primero se interpreta el signo y luego la influencia de las variables significativas. En términos matriciales, el modelo es el modo siguiente:

$\operatorname{Ln}\left(\frac{Y}{1-Y}\right)=\Sigma \beta X+u$

Donde $Y$ es un vector de variables binarias, de un valor igual a 1 cuando el individuo está sin empleo y busca trabajo, y de 0 cuando está empleado. $X$ es un conjunto de variables explicativas de redes sociales físicas, electrónicas, y variables de control. $\beta$ representa los coeficientes del modelo y $u$ es una perturbación no explicada por las variables independientes (Greene, 2003). En trabajos anteriores se usan especificaciones y variables similares (Martínez, 2015). Del modelo se desprenden tres hipótesis:

Hipótesis 1. Redes de amistad y desempleo. Se espera que las redes sociales físicas, en términos de cantidad de amigos, favorecerán que los individuos puedan obtener mayor información sobre vacantes apropiadas a sus capacidades y conocimientos, y que ello disminuya sus probabilidades de estar en situación de desempleo.

Hipótesis 2. Red familiary desempleo. Se espera que los familiares con disposición a apoyar a los individuos representen un efecto negativo en cuanto a la posibilidad de encontrarse desempleados.

Hipótesis 3. Redes sociales electrónicas en desempleo. Igualmente, se espera que tener cuentas en redes sociales electrónicas favorezca el intercambio de información entre empleados y desempleados. Ello beneficia a los buscadores de empleo para conocer características relevantes sobre el mercado laboral y posibles vacantes. Por lo cual se espera un impacto negativo del uso de redes sociales sobre la posibilidad de encontrarse desempleado.

\section{Descripción de las variables}

A continuación se describe la manera de construir la variable dependiente y las variables independientes.

Desempleo. La variable se construyó a partir de la situación laboral declarada por los individuos, tomando valores de 0 y 1 , donde 1 significa que el individuo se encuentra desempleado pero tiene disposición para encontrar empleo.

${ }^{7}$ Para más detalles del modelo logit, ver Greene (2003, cap. 21, secc. 7). 
Familia. A partir de la pregunta “¿Con cuántos familiares considera que siempre contará si se le presenta una urgencia o una necesidad?” se construyeron variables dummies para el número de familiares que pueden dar apoyo. Para los amigos se preguntó “ ¿Con cuántas personas que son no de su familia, considera que siempre contará si se le presenta una urgencia o una necesidad?". La variable de Facebook (face) es una variable dummy para quienes declararon estar registradas en esa red, igual se hizo con Twitter $(t w)$ y otra red social (ord $)^{8}$. También se construyó una variable para quienes pertenecen a cualquier red social electrónica ( $r s e$ ). Como variables de control se tomaron: entidad federativa (o estado), nivel educativo, sexo, tipo de empleo y grupo de edad.

\section{Datos}

Los datos provienen de la encuesta BIARE de 2014, realizada por el Instituto Nacional de Estadística y Geografía (INEGI). La encuesta ofrece la posibilidad de profundizar en la probabilidad de encontrar trabajo a partir de la cantidad de personas que integran el capital social de los individuos. En México, la mayoría de los estudios laborales incorporan datos de la Encuesta Nacional de Ocupación y Empleo (ENOE); infortunadamente dicha encuesta no ofrece información referente a la cantidad de amigos que los individuos poseen, dado lo cual la información para la estimación de los modelos fue obtenida de la BIARE.

La BIARE se basa en una muestra de 39.274 individuos, mayores de 18 años. Para las estimaciones del modelo la muestra es de 25.555 personas, ya que se toman los datos únicamente de las personas en situación de empleo o que buscan activamente uno. Aunque el enfoque principal de la primera encuesta refiere a características de bienestar subjetivo, ofrece información de situación laboral, ingresos salariales y cantidad de amigos y familiares con los que cuentan los individuos.

E1 cuadro 1 muestra algunos datos relevantes de las variables empleadas. Inicialmente se aprecia que en México el porcentaje de personas en situación de desempleo es baja. Ello en parte podría explicarse porque en el país no existe un seguro de desempleo gubernamental y por tanto el salario de reserva es bajo. Así, quienes enfrentan una situación de desempleo se ven en la necesidad de aceptar trabajo pronto, aunque la remuneración puede no ser la esperada. La estadística descriptiva muestra además que cerca del 30\% de los encuestados consideran que cuentan con más de 6 familiares que estarían dispuestos a apoyarlos en situaciones de urgencia. Ello confirma la idea de que en

${ }^{8}$ La BIARE no permite identificar si la otra red es Linkedin, Google plus, Myspace u otra. 
Latinoamérica los lazos familiares son muy fuertes. En referencia a la amistad, la tendencia no es muy clara; la cantidad de amigos que mayor porcentaje mostró fue 2, con un $21 \%$; esto es un indicador de que las redes de amistad pueden no ser tan extensas como las familiares. Sin embargo, el 14\% de los encuestados manifestaron que tenían 6 o más amigos que les podían apoyar en emergencias. E1 34\% de la población está inscrita en al menos una red social, siendo Facebook la más popular, con un 34\%, mientras Twitter cuenta con un 4,7\% y otras redes sociales con un $4,9 \%$. En el plano escolar se aprecia que más de la mitad de los encuestados tienen como nivel máximo primaria o secundaria (61\%), el $23 \%$ cuenta con nivel medio superior y el $16 \%$ posee un nivel de licenciatura o superior. E1 55\% de la muestra corresponde a personas de sexo femenino. Casi el 50\% se encuentran entre los 18 y los 39 años, y cerca del $11 \%$ son mayores de 65 . E1 63\% de los encuestados manifestó estar casado o en unión libre, el $20 \%$ soltero y el resto viudo, divorciado y otros. En promedio, los encuestados tenían 5 años de experiencia. En el apartado siguiente se describen los resultados.

Cuadro 1

Estadísticas descriptivas de las variables

\begin{tabular}{lccccc}
\hline Variable & Media & Desv. est. & Variable & Media & Desv. est. \\
\hline d_emp & 0,9545 & 0,2085 & d_Secundaria & 0,2636 & 0,4406 \\
d_bus_emp & 0,0455 & 0,2085 & d_Prepa & 0,1659 & 0,3720 \\
fam1 & 0,0925 & 0,2897 & d_Normal & 0,0108 & 0,1033 \\
fam2 & 0,1772 & 0,3818 & d_Tecnica & 0,0470 & 0,2116 \\
fam3 & 0,1732 & 0,3784 & d_prof_y_mas & 0,1636 & 0,3700 \\
fam4 & 0,1290 & 0,3352 & d_Mujer & 0,5584 & 0,4966 \\
fam5 & 0,0910 & 0,2876 & d_e18_24 & 0,1456 & 0,3527 \\
fam6 & 0,3092 & 0,4622 & d_e25_39 & 0,3458 & 0,4756 \\
amig1 & 0,1608 & 0,3674 & d_e40_54 & 0,2719 & 0,4449 \\
amig2 & 0,2147 & 0,4106 & d_e55_65 & 0,1276 & 0,3336 \\
amig3 & 0,1386 & 0,3456 & d_e66ymas & 0,1092 & 0,3119 \\
amig4 & 0,0685 & 0,2527 & d_soltero & 0,1967 & 0,3975 \\
amig5 & 0,0592 & 0,2360 & d_casado & 0,4515 & 0,4977 \\
amig6 & 0,1447 & 0,3518 & d_ulibre & 0,1757 & 0,3805 \\
Face & 0,3416 & 0,4743 & d_divorciado & 0,0272 & 0,1628 \\
Tw & 0,0465 & 0,2106 & d_viudo & 0,0730 & 0,2601 \\
Ord & 0,0485 & 0,2148 & Exp & 5,4333 & 9,1543 \\
d_Sin_educ & 0,0619 & 0,2410 & exp2 & 113,3197 & 284,8755 \\
d_Primaria & 0,2872 & 0,4525 & & & \\
\hline
\end{tabular}

Fuente: INEGI, BIARE 2014, elaboración propia.

\section{RESULTADOS}

Se plantearon diversas especificaciones, y en el documento se exponen las cuatro que mejores resultados mostraron (cuadro 2). Las regresiones consideran como variable dependiente el desempleo; en la primera se propone el total de amigos y familiares, en la segunda se dividen 
dichas variables para saber el impacto de cada amigo adicional hasta 6, en la última especificación se incorporan las variables correspondientes a redes sociales. En los modelos logit el signo del coeficiente es también el signo del efecto marginal (Cameron y Trivedi, 2005).

Cuadro 2

Determinantes del desempleo, México 2014

\begin{tabular}{|c|c|c|c|c|}
\hline Variables & Desempleo & Desempleo & Desempleo & Desempleo \\
\hline fam & $-0,008$ & & & \\
\hline amig & $-0,104^{* * *}$ & & & \\
\hline fam1 & & 0,059 & 0,058 & 0,058 \\
\hline fam2 & & $-0,038$ & $-0,040$ & $-0,038$ \\
\hline fam3 & & 0,059 & 0,057 & 0,059 \\
\hline fam4 & & 0,056 & 0,053 & 0,056 \\
\hline fam5 & & $-0,024$ & $-0,028$ & $-0,024$ \\
\hline fam6 & & $-0,027$ & $-0,031$ & $-0,027$ \\
\hline amig1 & & $-0,149$ & $-0,149$ & $-0,148$ \\
\hline amig 2 & & $-0,160^{*}$ & $-0,163^{*}$ & $-0,159^{*}$ \\
\hline amig3 & & $-0,356^{* * * *}$ & $-0,360^{* * * *}$ & $-0,355^{* * *}$ \\
\hline amig4 & & $-0,581^{* * *}$ & $-0,587^{* * *}$ & $-0,580^{* * * *}$ \\
\hline amig5 & & $-0,452^{* * * *}$ & $-0,464^{* * *}$ & $-0,451^{* * *}$ \\
\hline amig6 & & $-0,625^{* * *}$ & $-0,635^{* * * *}$ & $-0,625^{* * *}$ \\
\hline Face & & & ,037 & \\
\hline $\mathrm{Tw}$ & & & ,187 & \\
\hline Ord & & & 0,010 & \\
\hline Rse & & & & $-0,013$ \\
\hline d_Primaria & 0,052 & 0,046 & 0,047 & 0,046 \\
\hline d_Secundaria & 0,021 & 0,015 & 0,011 & 0,017 \\
\hline $\mathrm{d}^{-}$Prepa & 0,090 & 0,086 & 0,066 & 0,089 \\
\hline d_Normal & 0,000 & 0,000 & 0,000 & 0,000 \\
\hline d Tecnica & $-0,084$ & $-0,090$ & $-0,112$ & $-0,086$ \\
\hline d_prof_y_mas & 0,134 & 0,137 & 0,090 & 0,142 \\
\hline d Mujer & $-0,621^{* * * *}$ & $-0,622^{* * * *}$ & $-0,621^{* * * *}$ & $-0,621^{* * *}$ \\
\hline d_e25_39 & $-0,387^{* * *}$ & $-0,381^{* * * *}$ & $-0,369^{* * *}$ & $-0,383^{* * *}$ \\
\hline d_e40_54 & $-0,281^{* * *}$ & $-0,274^{* * *}$ & $-0,250^{* *}$ & $-0,278^{* * *}$ \\
\hline d_e55_65 & $-0,116$ & $-0,110$ & $-0,082$ & $-0,114$ \\
\hline d_e66ymas & $-0,693^{* * * *}$ & $-0,668^{* * * *}$ & $-0,642^{* * * *}$ & $-0,672^{* * *}$ \\
\hline d_casado & $-0,811^{* * * *}$ & $-0,814^{* * * *}$ & $-0,806^{* * * *}$ & $-0,814^{* * *}$ \\
\hline d_ulibre & $-0,758^{* * *}$ & $-0,764^{* * * *}$ & $-0,756^{* * *}$ & $-0,764^{* * *}$ \\
\hline d_divorciado & $-0,154$ & $-0,162$ & $-0,163$ & $-0,162$ \\
\hline d_viudo & $-0,624^{* * *}$ & $-0,604^{* * * *}$ & $-0,602^{* * * *}$ & $-0,605^{* * *}$ \\
\hline Exp & $-0,0485^{* * *}$ & $-0,0486^{* * * *}$ & $-0,0492^{* * *}$ & $-0,0485^{* * *}$ \\
\hline $\exp 2$ & $0,000773 *$ & $0,001^{*}$ & $0,000779^{*}$ & $0,000757^{*}$ \\
\hline $\begin{array}{l}31 \text { dummies } \\
\text { para estados }\end{array}$ & $\mathrm{x}$ & $\mathrm{x}$ & $\mathrm{x}$ & $\mathrm{x}$ \\
\hline _cons & $-1,362^{* * *}$ & $-1,390^{* * *}$ & $-1,416^{* * *}$ & $-1,387^{* * *}$ \\
\hline$\overline{\mathrm{N}}$ & 25.152 & 25.311 & 25.311 & 25.311 \\
\hline $\mathrm{R}^{2}$ & 0,062 & 0,062 & 0,062 & 0,062 \\
\hline $\begin{array}{l}\text { red social electroni } \\
\text { y } 4 \text { de grupo de ed } \\
\text { Fuentes: INEGI. }\end{array}$ & $\begin{array}{l}\text { lica; d_Mujer si } \\
\text { dad. } \\
\text { BIARE } 2014 .\end{array}$ & $\begin{array}{l}\text { n variable } \\
\text { o de no f } \\
\text { está regist } \\
\text { o es muje }\end{array}$ & $\begin{array}{l}\text { Inúmero } \\
\text { apoyan al } \\
\text { r; Ord re } \\
\text { mies de e }\end{array}$ & $\begin{array}{l}\text { s que apoyan } \\
\text { face indica s } \\
\text { a red social; } R \\
\text { nivel educat }\end{array}$ \\
\hline
\end{tabular}


Los análisis realizados aciertan un $95 \%$ de los resultados, con un pseudo $\mathrm{R}^{2}$ de 0,06, similar al de trabajos anteriores (Martínez, 2015). Si bien las variables disponibles explican en niveles bajos la posibilidad de desempleo, se puede considerar que el modelo es aceptable por el hecho de tener un porcentaje elevado de aciertos. Los resultados de la primera regresión sugieren que la cantidad de familiares dispuestos a apoyar no tiene un efecto significativo sobre las probabilidades de que un individuo se encuentre desempleado. A pesar de que ello pudiera resultar contraintuitivo, es posible dar una explicación: la familia es una institución que puede ofrecer al individuo compartir alimentos y bienes duraderos. Gracias a ello los desempleados no se ven en la necesidad de disminuir sus salarios de reserva. El apoyo familiar podría constituirse en una especie de "seguro de desempleo" en el caso de México. De esta manera, los resultados rechazan la primera hipótesis planteada en el estudio.

En lo que respecta a los amigos se da un caso diferente; dicha variable resulta significativa y representa un signo negativo, es decir, la cantidad de amigos que un individuo posee disminuye sus probabilidades de estar en situación de desempleo. A partir de tres amigos existe una menor probabilidad de que los individuos se encuentren en situación de desempleo, esto es, más amigos implica mayores probabilidades de estar trabajando. Ello es el resultado esperado de acuerdo a la revisión de la literatura y la evidencia empírica. En el caso de los amigos es difícil que estos cumplan una función de seguro de desempleo, sin embargo, sí pueden compartir información sobre vacantes disponibles que pudieran ser de interés para los desempleados, ya que es probable que tengan niveles de calificación similar. Así el capital social también resulta en capital informativo que les ofrece a quienes buscan empleo mayor disponibilidad de datos relevantes sobre el mercado laboral. De esta manera las variables referentes a amigos respaldaron la segunda hipótesis. Las redes de amistad contribuyen a que exista menor posibilidad de desempleo para los individuos.

En lo que respecta a las redes sociales electrónicas ninguna de las tres variables resultó significativa ${ }^{9}$. Si bien este tipo de redes permite estar en contacto con familiares y amigos, no es significativo como medio de información sobre el mercado laboral.

Los resultados sugieren que una amplia red de amigos dispuestos a apoyar a los individuos en situaciones de urgencia disminuye significativamente la probabilidad de encontrarse en situación de desempleo; no ocurre lo mismo con la red familiar y ni participar en redes sociales electrónicas.

${ }_{9}^{9}$ No hay una correlación fuerte entre redes físicas y redes electrónicas. 
En el cuadro 3 se muestran los efectos marginales de las estimaciones realizadas. La primera estimación asume una contribución constante de cada familiar y amigo. Al observar los efectos marginales se aprecia que cada amigo reduce en un 0,4\% la probabilidad de encontrarse en situación de desempleo. Sin embargo esta lectura no es del todo apropiada porque la pregunta sobre el número de no familiares que dan apoyo se refiere a 0-5 personas y a más de 6; de modo que no es posible determinar el impacto de tener más de 6 amigos.

Cuadro 3

Efectos marginales sobre el desempleo, México 2014

\begin{tabular}{|c|c|c|c|c|}
\hline Variables & Desempleo & Desempleo & Desempleo & Desempleo \\
\hline fam & 0,0000 & & & \\
\hline amig & $-0,004^{* * * * *}$ & & & \\
\hline fam1 (d) & & 0,0020 & 0,0020 & 0,0020 \\
\hline fam $2(d)$ & & $-0,0010$ & $-0,0010$ & $-0,0010$ \\
\hline fam $3(d)$ & & 0,0020 & 0,0020 & 0,0020 \\
\hline fam 4 (d) & & 0,0020 & 0,0020 & 0,0020 \\
\hline fam5 (d) & & $-0,0010$ & $-0,0010$ & $-0,0010$ \\
\hline fam6 (d) & & $-0,0010$ & $-0,0010$ & $-0,0010$ \\
\hline $\operatorname{amig} 1(\mathrm{~d})$ & & $-0,0050$ & $-0,0050$ & $-0,0050$ \\
\hline $\operatorname{amig} 2(d)$ & & $-0,0050$ & $-0,0050$ & $-0,0050$ \\
\hline amig3 (d) & & $-0,011^{\text {**** }}$ & $-0,011^{* * * *}$ & $-0,011^{\text {***** }}$ \\
\hline amig 4 (d) & & $-0,016^{* * * *}$ & $-0,016^{* * * *}$ & $-0,016^{* * *}$ \\
\hline amig5 (d) & & $-0,013^{* * * *}$ & $-0,014^{* * * * *}$ & $-0,013^{\text {**** }}$ \\
\hline amig6 (d) & & $-0,018^{\text {***** }}$ & $-0,018^{\text {***k*k }}$ & $-0,018^{\text {**** }}$ \\
\hline face $(d)$ & & & 0,0010 & \\
\hline tw $(d)$ & & & 0,0070 & \\
\hline ord (d) & & & 0,0000 & \\
\hline rse (d) & & & & 0,0000 \\
\hline d_Primaria ) & 0,0020 & 0,0020 & 0,0020 & 0,0020 \\
\hline d_Secundar ) & 0,0010 & 0,0010 & 0,0000 & 0,0010 \\
\hline d_Prepa (d) & 0,0030 & 0,0030 & 0,0020 & 0,0030 \\
\hline d_Normal & & & & \\
\hline d_Tecnica ) & $-0,0030$ & $-0,0030$ & $-0,0040$ & $-0,0030$ \\
\hline d_prof_y_m ) & 0,0050 & 0,0050 & 0,0030 & 0,0050 \\
\hline d_Mujer (d) & $-0,021^{\text {****** }}$ & $-0,021^{\text {****** }}$ & $-0,021^{* * * * *}$ & $-0,021^{\text {****** }}$ \\
\hline d_e25_39 (d) & $-0,013^{\text {***** }}$ & $-0,013^{\text {***** }}$ & $-0,013^{* * * *}$ & $-0,013^{* * * *}$ \\
\hline d_e40_54 (d) & $-0,009^{\text {*** }}$ & $-0,009^{* *}$ & $-0,008^{*}$ & $-0,009^{* * *}$ \\
\hline d $e 5565(d)$ & $-0,0040$ & $-0,0040$ & $-0,0030$ & $-0,0040$ \\
\hline d_e66ymas ) & $-0,018^{* * * *}$ & $-0,018^{* * * *}$ & $-0,017^{* * * * *}$ & $-0,018^{* * * *}$ \\
\hline d_casado (d) & $-0,028^{* * * *}$ & $-0,028^{* * * *}$ & $-0,028^{* * * * *}$ & $-0,028^{* * * *}$ \\
\hline d_ulibre (d) & $-0,022^{* * * *}$ & $-0,022^{\text {****k }}$ & $-0,022^{\text {***** }}$ & $-0,022^{* * * * *}$ \\
\hline d_divorcia ) & $-0,0050$ & $-0,0050$ & $-0,0050$ & $-0,0050$ \\
\hline d_viudo $(\mathrm{d})$ & $-0,017^{* * * *}$ & $-0,017^{* * * * *}$ & $-0,017^{* * * * *}$ & $-0,017^{* * * * *}$ \\
\hline $\exp$ & $-0,002^{* * * *}$ & $-0,002^{\text {***** }}$ & $-0,002^{\text {****** }}$ & $-0,002^{* * * * *}$ \\
\hline $\exp 2$ & 0,0000 & 0,0000 & 0,0000 & 0,0000 \\
\hline 31 dummies de & $\mathrm{x}$ & $\mathrm{x}$ & $\mathrm{x}$ & $\mathrm{x}$ \\
\hline
\end{tabular}

Fuente: INEGI, BIARE 2014, elaboración propia.

Para afinar ese aspecto se estimaron regresiones que consideraban amigo por amigo. A partir de ello se observa nuevamente que los 
familiares no representan una buena opción para encontrar empleo; una mayor red de apoyo familiar no significa menores probabilidades de estar en situación de desempleo. En lo referente a redes de amistad se aprecia que, al modificar la especificación, tener uno o dos amigos tampoco tiene un impacto en la obtención de un trabajo. Sin embargo, tener 3, 4 o 5 amigos contribuye entre un 1,1\% y un 1,6\% en la probabilidad de tener empleo. Contar con 6 o más personas no familiares dando apoyo representa un incremento en la probabilidad de estar empleado del 1,8\%.

En la tercera especificación se mantienen los efectos marginales de las redes físicas de amistad y familia. En dicha estimación se incorporan las variables referentes a redes sociales electrónicas. Ahí se observa que no existe un efecto significativo entre estar inscrito en este tipo de plataformas de amistad y contar con un empleo.

Además se evaluaron otras variables relevantes de las características de los individuos. Ningún nivel educativo implica mayores probabilidades de estar desempleado. Las mujeres tienen $2,1 \%$ más probabilidades de estar empleadas. El grupo de edad con menor probabilidad de desempleo es el de personas mayores de 65 años, con un 1,8\%. Estar casado aumenta en un 3\% la posibilidad de tener empleo, mientras que estar en unión libre o viudo la aumenta en un 1,9 y un 2,2\%; ser divorciado no tuvo significancia estadística. Y un año más de experiencia laboral implica una probabilidad 0,2\% mayor de estar empleado.

Los modelos de esta sección muestran algunas ventajas de tener una amplia red de contactos. E1 apoyo familiar no incide negativamente en la posibilidad de estar desempleado. Los amigos favorecen la posibilidad de encontrar empleo porque proporcionan información relevante sobre el mercado y pueden hacer recomendaciones a sus empleadores; pueden proporcionar información de vacantes que no está disponible en las oficinas públicas ni en internet, y simplificar la búsqueda laboral.

\section{Discusión}

La investigación confirmó algunas de las hipótesis propuestas. Las redes sociales físicas favorecen que los individuos consigan un empleo. De esta manera, no hay evidencia estadística para rechazar la primera hipótesis: los contactos personales son de ayuda para obtener empleo en el caso de personas no familiares y únicamente si la red supera los tres amigos.

En cuanto al soporte social, las estimaciones mostraron que las personas que cuentan con más de tres amigos tienen menores proba- 
bilidades de estar desempleadas. La pregunta utilizada apunta a las personas ajenas a la familia del individuo que lo apoyarían siempre en una urgencia o necesidad; dicho cuestionamiento iría encaminado a relaciones sociales sólidas. Sin embargo, las personas no solo tienen relaciones sólidas, también cuentan con relaciones superficiales que no involucran lazos afectivos profundos. Cabe señalar que tanto los amigos cercanos como los lejanos pueden ser fuente de información sobre el mercado laboral. Aquellos individuos que cuentan con una elevada cantidad de amigos cercanos es probable que también posean una amplia red de contactos. De esta manera, las personas muy sociables dispondrán de un mayor acceso a información sobre vacantes y opciones laborales. Asimismo, es frecuente que los integrantes de la red de contactos y amigos de un individuo tengan un nivel educativo o de experiencia similar a este y estén integrados laboralmente en sectores y actividades atractivas para el desempleado.

La red familiar no muestra una contribución significativa para que los individuos obtengan un empleo, con lo que se vio rechazada la segunda hipótesis. La razón de esto puede ser que los familiares no necesariamente tienen la misma preparación que la persona desempleada, luego las opciones laborales que pueden comunicar no empatan adecuadamente con la búsqueda del desocupado. Otra causa puede ser que el apoyo de parientes se encamine a aspectos ajenos al mercado laboral; puede ocurrir que padres y hermanos brinden ayuda al desempleado en términos pecuniarios. Los individuos que observen a su familiar en situación de desempleo pueden ayudarle compartiendo alimentos o apoyándolo en lo que se refiere a gastos personales o del hogar. De esta manera, el soporte familiar le permite al desempleado mexicano la posibilidad de no reducir su salario de reserva. Gracias al apoyo de la familia, el buscador de empleo tiene la posibilidad de rechazar ofertas laborales que no empaten con sus capacidades y competencias. Lo que significa un periodo mayor en situación de desempleo.

La tercera hipótesis estuvo enfocada en las redes sociales electrónicas y las estimaciones no la respaldan. Los datos muestran que estar inscrito en Facebook no ejerce un efecto significativo en la posibilidad de encontrarse desempleado; asimismo pertenecer a Twitter o a otras redes sociales no resulta significativo. Si bien el uso de medios electrónicos para comunicarse con familiares y amigos puede permitir a los individuos mantener lazos afectivos, también puede ser un distractor. Los lazos establecidos en redes sociales electrónicas son más débiles que aquellos establecidos en la convivencia real y cotidiana. Así, 
comunicarse con amigos y familiares a través de medios electrónicos no implica necesariamente una cercanía emocional. Las actividades de índole social pueden representar en los buscadores de empleo un uso inadecuado del tiempo.

Recurrir a personas al interior de las empresas para aproximar empleadores y desempleados es un esquema que permite a unos y otros acceder a información que los medios formales no proveen. El empleador puede indagar con el enlace sobre características relevantes del aspirante a ocupar la posición. Puede informarse sobre antecedentes laborales, competencias profesionales, habilidades particulares, incluso antes de empezar los procesos de entrevistas. De esta manera el empleador puede reducir el costo de búsqueda asociado al proceso de cubrir vacantes en una firma. A los desempleados les permite saber si el empleo en cuestión es conveniente para ellos, en términos de salarios, prestaciones que otorgan las empresas, actitudes de los posibles compañeros de trabajo o de los superiores, etc. De esta manera, contar con un enlace mejora la calidad del emparejamiento entre desempleados y vacantes disponibles.

\section{CONCLUSIONES}

Este trabajo muestra que, tal como sugiere la teoría económica, el capital social de un individuo favorece la consecución de empleo. Las redes sociales físicas permiten a las personas obtener información sobre el mercado laboral, empresas y vacantes específicas. De esta manera los individuos llegan mejor preparados a encarar entrevistas laborales y tienen mayores posibilidades de obtener empleo.

Dentro de los resultados cabe destacar que la red familiar no contribuye a la posibilidad de obtener empleo. El resultado puede interpretarse en el sentido de que la familia puede jugar el papel del inexistente seguro de desempleo para los mexicanos. Se plantea que los parientes brindan apoyo a los buscadores de trabajo y les permiten cubrir ciertas necesidades, les otorgan la oportunidad de buscar un empleo con mayor paciencia que si carecieran de una red de ayuda.

Al igual que con la red familiar, pertenecer a redes sociales electrónicas no ejerce un efecto positivo en la obtención de un empleo. Sin embargo, ello es un indicador de que los mejores esquemas para buscar empleo en línea pasan por medios como los portales de empleo del gobierno, empresas o agencias de colocación.

El presente trabajo se distingue de otros estudios que vinculan capital social y mercado laboral en varios aspectos. El enfoque de la investigación se concentra en la condición de empleo de los indivi- 
duos encuestados, esto gracias a que en México se cuenta con una reciente encuesta que incorpora el soporte social y familiar que posee un individuo, su pertenencia a redes sociales virtuales y su condición laboral. De esta manera fue posible profundizar en los posibles impactos generados por el capital social con el que cuentan los mexicanos.

Asimismo, a partir de la información disponible otras investigación han permitido indagar en las implicaciones salariales relacionadas con una red social y familiar amplia (Charles-L. et al., s.f.).

$\mathrm{E} 1$ presente artículo no indaga en la duración del desempleo o en la calidad del trabajo obtenido a partir de las relaciones de amistad o familia. Los datos tampoco permiten abordar la calidad del empleo de amigos y familiares de los individuos, por lo que no fue posible hacer una exploración sobre el tema. Precisamente este podría ser un tema de investigación para futuras investigaciones académicas.

\section{REFERENCIAS BIBLIOGRÁFICAS}

Aguilera, M. B. (2002). The impact of social capital on labor force participation: Evidence from the 2000 Social Capital Benchmark Survey. Social Science Quarterly, 83(3), 853-874.

Aguilera, M. B. y Massey, D. S. (2003). Social capital and the wages of mexican migrants: New hypotheses and tests. Social Forces, 82(2), 671-701.

Bachmann, R. y Baumgarten, D. (2012). How do the unemployed search for a job? Evidence from the EU Labour Force Survey. IZA Discussion Paper, 6753, 1-28.

Becker, G. S. (1962). Investment in human capital: A theoretical analysis. The Journal of Political Economy, 7O(5), 9-49.

Becker, G. S. (1981). Altruism in the family and selfishness in the market place. Economica, 48(189), 1-15.

Bentolila, S., Michelacci, C. y Suárez, J. (2003). Social networks and occupational choice, mimeo. Centre for Economic Performance. London School of Economics.

Calvó-A., A. e Ioannides, Y. M. (2005). Social networks in labor markets. Discussion paper, Departament of Economics, Tufts University.

Calvó-A., A. y Jackson, M. O. (2004). The effect of social networks on employment and inequality. The American Economic Review, 94(3), 426-454.

Cameron, A. C. y Trivedi, P. K. (2005). Microeconometrics. Methods and applications. Cambridge, UK., Nueva York: Cambridge University Press.

Cappellari, L. y Tatsiramos, K. (2011). Friends' networks and job finding rates. CESifo working paper series 3243.

Charles-L., H., Torres, A. y Castro, D. (s. f.). Efectos del capital social en los salarios en México (en arbitraje).

Charles-L., H. (s.f.). Un análisis exploratorio sobre redes sociales y empleo. E1 caso de México (en arbitraje). 
Diamond, P. A. (1982). Aggregate demand management in search equilibrium. Journal of Political Economy, 90(5), 881-894.

Ellison, N. B., Steinfield, C. y Lampe, C. (2007). The benefits of Facebook "friends": Social capital and college students' use of online social network sites. Journal of Computer-Mediated Communication, 12(4), 1143-1168.

Greene, W. (2003). Econometric analysis, 5. ${ }^{a}$ ed. Upper Saddle River, NJ: Prentice Hall.

Helliwell, J. F. y Putnam, R. D. (2004). The social context of well-being. Philosophical Transactions of the Royal Society of London. Series B, Biological Sciences, 359(August), 1435-1446.

Ioannides, Y., Overman, H., Rossi-H. et al. (2008). The effect of information and communication technologies on urban structure. Economic Policy, 23(54), 201-242.

Kugler, A. D. (2003). Employee referrals and efficiency wages. Labour Economics, 10(5), 531-556.

Kuhn, P. y Mansour, H. (2014). Is internet job search still ineffective? Economic Journal, 124, 1213-1233.

Kwak, H., Lee, C., Park, H. et al. (2010). What is Twitter, a social network or a news media? Categories and subject descriptors. En Proceedings of the 19th International Conference on World Wide Web (pp. 591-600), [http://doi.org/http://doi.acm.org/10.1145/1772690.1772751].

Lin, M. (2008). Does unemployment increase crime? Journal of Human Resources, 43(2), 413-436.

Mang, C. (2012). Online Job Search and Matching Quality. IFO working papers $147,1-34$.

Martínez, S. (2015). Determinantes del desempleo. Valladolid: Universidad de Valladolid.

Montgomery, J. D. (1991). Social networks and labor-market outcomes: Toward an economic analysis. The American Economic Review, 81(5), 1408-1418.

Mortensen, D. (1982). The matching process as a noncooperative bargaining game. En J. J. McCall (ed.), The Economics of Information and Uncertainty (pp. 233-258). Chicago y Londres: University of Chicago Press.

Pénard, T., Poussing, N. y Suire, R. (2013). Does the internet make people happier? Journal of Socio-Economics, 46(2013), 105-116.

Petrongolo, B. y Pissarides, C. (2001). Looking into the black box: A survey of the matching function. Journal of Economic Literature, 39(2), 390-431.

Rees, A. (1966). Labor economics: Effects of more knowledge. American Economic Review, 56(1), 559-566.

Rees, A. y Schultz, G. P. (1970). Workers and wages in an urban labor market. Chicago y Londres: University of Chicago Press.

Reid, G. L. (1972). Job search and the effectiveness of job-finding methods. Industrial and Labor Relations Review, 25(4), 479-495.

Stigler, G. J. (1962). Information in the labor market. Journal of Political Economy, 70(5), 94-105. 
Torres, A. y Félix, G. (2017). El uso de internet y su relación con los salarios en México: un análisis no paramétrico. En G. Ochoa y A. Torres (coords.), Los retos del cambio económico actual: revisión y aplicaciones para el caso mexicano (pp. 31-50). Monterrey: Ediciones de Laurel.

Valenzuela, S., Park, N. y Kee, K. F. (2009). Is there social capital in a social network site?: Facebook use and college student's life satisfaction, trust, and participation. Journal of Computer-Mediated Communication, 14(4), 875-901.

Wial, H. (2008). Getting a good job: Mobility in a segmented labor market. Industrial Relations: A Journal of Economy and Society, 3O(3), 396-416.

Winkelmann, L. y Winkelmann, R. (1998). Why are the unemployed so unhappy? Evidence from panel data. Economica, 65(257), 1-15. 